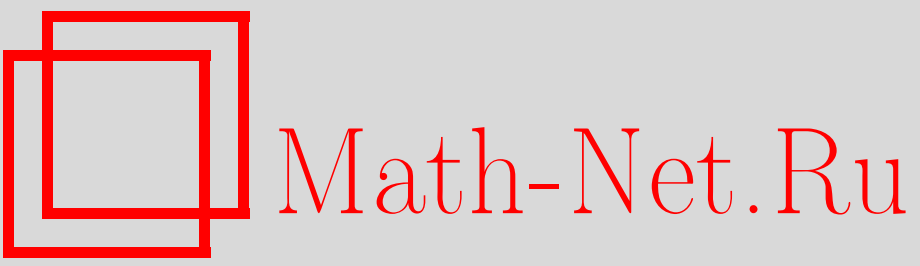

С. С. Рышков, Прямое геометрическое описание $n$-мерных параллелоэдров второго типа Вороного, УМН, 1999, том 54, выпуск 1, 263-264

DOI: https://doi.org/10.4213/rm129

Использование Общероссийского математического портала Math-Net.Ru подразумевает, что вы прочитали и согласны с пользовательским соглашением

http://www . mathnet.ru/rus/agreement

Параметры загрузки:

IP : 54.157 .27 .8

26 апреля 2023 г., 12:19:40 


\title{
ПРЯМОЕ ГЕОМЕТРИЧЕСКОЕ ОПИСАНИЕ $n$-МЕРНЫХ ПАРАЛЛЕЛОЭДРОВ ВТОРОГО ТИПА ВОРОНОГО
}

\author{
С. С. Рышков
}

$0^{\circ}$. В заметке [1] изучались $n$-мерные параллелоэдры. В ней, в частности, исследование всех примитивных $n$-мерных параллелоэдров было сведено к исследованию параллелоэдров нового класса: "коренных параллелоэдров", были приведены примеры таких параллелоэдров, дано прямое геометрическое описание произвольного $n$-мерного параллелоэдра первого типа Вороного, а также всех четырехмерных параллелоэдров.

Была анонсирована теорема 1:

Для чисел $n_{1}=1, n_{2}=4, n_{3}=5, \ldots, n_{k}=k+2, \ldots$ в евклидовьх пространствах $\mathbb{E}^{n_{k}}$ определень, вообще говоря, не примитвные параллелоэдры (которые названь коренными), удовлетворяющ,ие следующим условиям:

1) при каждом натуральном $n$ для любого п-мерного примитивного параллелоэдра $\mathscr{P} \subset \mathbb{E}^{n}$ найдется не менее чем $n(n+1) / 2$ коренных параллелоэдров $\mathscr{P}_{1}, \mathscr{P}_{2}, \ldots, \mathscr{P}_{\sigma}$, имеющих размерность, не превосходящую $n$, и расположенных в $\mathbb{E}^{n}$ таким образом, что

$$
\mathscr{P}=\lambda_{1} \mathscr{P}_{1} \oplus \lambda_{2} \mathscr{P}_{2} \oplus \cdots \oplus \lambda_{\sigma} \mathscr{P}_{\sigma},
$$

где через $\oplus$ обозначено векторное сложение (суммирование по Минковскому), а $\lambda_{1}, \lambda_{2}$, $\ldots, \lambda_{\sigma}$ w- положительнье числа;

2) каждый п-мерный примитивный параллелоэдр $\mathscr{P}^{\prime} \subset \mathbb{E}^{n}$ того же типа, что и $\mathscr{P}$ представим (с точностью до движения параллелоәдра $\mathscr{P}^{\prime}$ в пространстве $\mathbb{E}^{n}$ и аффинного преобразования всего пространства $\left.\mathbb{E}^{n}\right)$ в виде суммы (*), но, быть может, с другим набором чисел $\lambda_{1}, \lambda_{2}, \ldots, \lambda_{\sigma}$.

$1^{\circ}$. В предлагаемой заметке для каждого $n \geqslant 4$ приводится $\left(3^{\circ}\right)$ в качестве еще одного примеpa $n$-мерного коренного параллелоэдра некоторый многогранник $\bar{M}_{n}$.

На основе многогранника $\bar{M}_{n}$ дается $\left(4^{\circ}\right)$ простое геометрическое описание (при любом $n \geqslant 4)$ произвольного параллелоэдра второго типа Вороного.

Обсуждается $\left(5^{\circ}\right)$ вопрос о "толщине" области Вороного-Дирихле в данном направлении.

Ставится ряд задач $\left(6^{\circ}\right)$ различного характера и, по-видимому, различной трудности.

$2^{\circ}$. Обозначим через $\mathbb{Q}^{n} \subset \mathbb{E}^{n}$ некоторый куб. Дуальный к нему многогранник, т.е. выпуклое замыкание $\mathbb{O}^{n}$ совокупности центров всех $(n-1)$-мерных граней куба $\mathbb{Q}^{n}$, мы называем $n$-мерным правильным ортаәдром). (Очевидным способом определяются аффинный и комбинаторный ортаэдры.) Общий центр многогранников $\mathbb{Q}^{n}$ и $\mathbb{O}^{n}$ обозначим через $C$.

В каждом $n$-мерном ортаэдре естественно выделяются $n$ пар взаимно противоположных вершин. В правильном и афффинном ортаэдре каждой такой паре отвечает $(n-1)$-мерный $\partial и а м е-$ тральный ортаэдр - выпуклая оболочка всех вершин $n$-мерного ортаэдра, кроме вершин из этой пары.

Пусть ортаэдр $\mathbb{Q}^{n}$ имеет центр в начале координат, а его вершины лежат в точках $\pm e_{i}$. Тогда каждому вектору $\pm\left(e_{i} \pm e_{j}\right)$ естественно ставится в соответствие пара ребер ортаэдра $\mathbb{Q}^{n}$, взятых без направления. Рассмотрим набор всех векторов вида $e_{i}+e_{j}$, где $i, j=1,2, \ldots, n, i \neq j$, и множество $\mathscr{D}$ всех отвечающих этому набору ребер ортаэдра $\mathbb{Q}^{n}$.

Назовем антисимплициальнылм подграфом реберного графа (1-мерного скелета) правильного ортаэдра $\mathbb{Q}^{n}$ любой такой его подграф, который получается из множества $\mathscr{D}$ произвольным совмещением ортаэдра $\mathbb{Q}^{n}$ с собой.

$3^{\circ}$. Рассмотрим вторую совершенную форму (т.е. форму $V_{n}$ по Коркину и Золотареву [2], $\phi_{1}^{(n)}$ по Вороному [3], или $D_{n}$ по Коксетеру [4]). Обозначим через $\bar{M}_{n}$ область Вороного-Дирихле решетки $\Gamma$, соответствуюшей форме, взаимной к $V_{n}$. Как область Вороного-Дирихле решетки, многогранник $\bar{M}_{n}$ есть параллелоэдр.

Работа выполнена при поддержке Российского фонда фундаментальных исследований (грант № 96-01-00166). 
Лемма 1. С точностью до движения и подобия многогранник $\bar{M}_{n}$ совпадает с многогранником $\mathbb{Q}^{n} \cap \mu \mathbb{O}^{n}$, где $\mu \mathbb{O}^{n}$ - многогранник гомотетичный ортаэдру $\mathbb{O}^{n}$ с коэффициентом гомотетии $\mu=n / 2$ и центром гомотетии в точке $C$.

(Лемма доказьвается констуктивно с использованием геометрической структуры решетки $Г$.)

$4^{\circ}$. Пусть теперь $L-$ произвольный антисимплициальный подграб̆ реберного граффа произвольного диаметрального ортаэдра исходного ортаэдра $\mathbb{O}^{n}$ (имеющий $(n-1)(n-2)$ ребер).

Обозначим через $R_{n}$ любое такое подмножество ребер реберного графа ортаэдра $\mathbb{O}^{n}$, которое не содержит ребер из $L$, а из каждых двух параллельных ребер ортаэдра $\mathbb{O}^{n}$, разумеется, не лежащих в $L$, содержит только одно.

В нижес ледующей теореме под словами "параллелоэдр второго типа" понимается быть может и вырожденная область Вороного-Дирихле решетки второго типа.

ТеОРема 1. Для любого $n \geqslant 4$ многогранник $\bar{M}_{n}$ является коренным параллелоэдром.

Каждый n-мерный параллелоәдр $\mathscr{P} \subset \mathbb{E}^{n}$ второго типа представим (с точностью до движения параллелоэдра $\mathscr{P}$ в пространстве $\mathbb{E}^{n}$ и аффинного преобразования всего пространства $\left.\mathbb{E}^{n}\right)$ в виде сумми $(*)$, где $\mathscr{P}_{1}=\bar{M}_{n}$, а $\mathscr{P}_{2}, \mathscr{P}_{3}, \ldots, \mathscr{P}_{\sigma}-$ ребра графа $R_{n}$. (В случае вырожденного параллелоэдра некоторые из чисел $\lambda_{1}, \lambda_{2}, \ldots, \lambda_{\sigma}$ равны нулю.)

Обратим внимание, что мноогогранники $M_{4}$, см. [1], и $\bar{M}_{4}$ с точностью до движения и подобия совпадают между собой и являются правильными 24 -гранниками в пространстве $\mathbb{E}^{4}$.

$5^{\circ}$. Пусть $\mathbb{P} \subset \mathbb{E}^{n}$ - замкнутое выпуклое тело и $\mathbb{E}^{k} \subset \mathbb{E}^{n}$, где $0<k<n$, фиксированная плоскость. Говорят [5], что тело $\mathbb{P}$ имеет ненулевую толщину в направлении $\mathbb{E}^{k}$, если его пересечение с произвольной плоскостью $\mathbb{E}_{1}^{k}$, параллельной плоскости $\mathbb{E}^{k}$, либо пусто, либо $k$-мерно.

Следующая теорема достаточно очевидна, кроме того она срвзу вытекает из теоремы 2 работы Б. А. Венкова [5], а также из исследований работы [1].

Теорема 2. Для того чтобъ параллелоэдр $\mathscr{P}^{n} \subset \mathbb{E}^{n}$, имел ненулевую толщину в направлении $\mathbb{E}^{1} \subset \mathbb{E}^{n}$ необходимо и достаточно, чтобы $\mathscr{P}^{n}=\mathscr{P}^{l} \oplus \mathscr{P}^{1}$, əде $n-1 \leqslant l \leqslant n u \mathscr{P}^{1} \subset \mathbb{E}^{1}$.

В формулировке этой теоремы мы под словом $r$-мерный параллелоэдр, где $r$ принимает указьваемые значения, понимаем параллелоэдр $\left(\mathscr{P}^{r} \subset \mathbb{E}^{r} \subset \mathbb{E}^{n}\right)$, являющийся областью Вороного-Дирихле $r$-мерной решетки.

$6^{\circ}$. ПроБЛЕмы.

1. Найти все коренные 5-мерные параллелоэдры. Дать описание всех типов и областей типов 5-мерных параллелоэдров через коренные.

2. Найти подход к описанию (алгоритм разыскания) всех $n$-мерных коренных параллелоэдров.

3. Верно ли обобщение теоремы 2 на $k$-мерное направление?

\section{СПИСОК ЛИТЕРАТУРЫ}

[1] Рышков С. С. // УМН. 1998. Т. 53. № 2. С. 161-162. [2] Вороной Г. Ф. // Собр. сочинений. Т. 2. Киев: Изд-во АН УССР, 1952. С. 239-368. [3] Korkine A., Zolotareff G. // Math. Ann. 1877. V. 11. Р. 242-292 (см. также Е. И. Золотарев, Полное собр. сочинений, вып. 1, Изд-во АН, 1931, С. 375-434). [4] Coxeter H. S. M. // Canad. J. Math. 1951. V. 3. P. 391-441. [5] Венков Б. А. // Избранные труды. Л.: Наука, 1981. С. 362-379 (оригинал в Матем. сб., 1959, T. 49, № 2, C. 207-224). 